ARTICLE

\title{
Two distinct superconducting states controlled by orientations of local wrinkles in LiFeAs
}

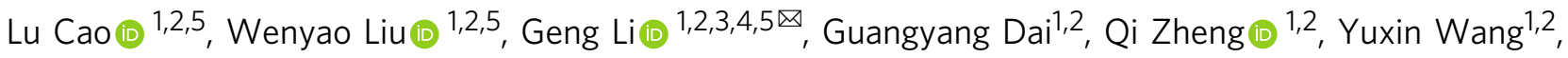
Kun Jiang1,2, Shiyu Zhu (1) 1,2, Li Huang1,2,3, Lingyuan Kong (10 1, Fazhi Yang1,2, Xiancheng Wang 1,3,4,

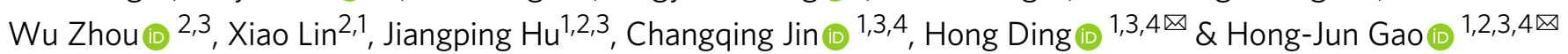

For iron-based superconductors, the phase diagrams under pressure or strain exhibit emergent phenomena between unconventional superconductivity and other electronic orders, varying in different systems. As a stoichiometric superconductor, LiFeAs has no structure phase transitions or entangled electronic states, which manifests an ideal platform to explore the pressure or strain effect on unconventional superconductivity. Here, we observe two types of superconducting states controlled by orientations of local wrinkles on the surface of LiFeAs. Using scanning tunneling microscopy/spectroscopy, we find type-I wrinkles enlarge the superconducting gaps and enhance the transition temperature, whereas type-II wrinkles significantly suppress the superconducting gaps. The vortices on wrinkles show a $\mathrm{C}_{2}$ symmetry, indicating the strain effects on the wrinkles. By statistics, we find that the two types of wrinkles are categorized by their orientations. Our results demonstrate that the local strain effect with different directions can tune the superconducting order parameter of LiFeAs very differently, suggesting that the band shifting induced by directional pressure may play an important role in iron-based superconductivity.

\footnotetext{
${ }^{1}$ Institute of Physics, Chinese Academy of Sciences, Beijing 100190, China. ${ }^{2}$ School of Physical Sciences, University of Chinese Academy of Sciences, Beijing 100049, China. ${ }^{3}$ CAS Center for Excellence in Topological Quantum Computation, University of Chinese Academy of Sciences, Beijing 100190, China.

${ }^{4}$ Songshan Lake Materials Laboratory, Dongguan, Guangdong 523808, China. ${ }^{5}$ These authors contributed equally: Lu Cao, Wenyao Liu, Geng Li.

凶email: gengli.iop@iphy.ac.cn; dingh@iphy.ac.cn; hjgao@iphy.ac.cn
} 
$\mathrm{T}$ he origin of superconductivity in iron-based superconductors (FeSCs) remains elusive despite intensive research efforts over a dozen years ${ }^{1-3}$. The large orbital degrees of freedom, as well as the presence of intertwining orders hinder a microscopic understanding of the pairing mechanism in FeSCs (refs. ${ }^{4-6}$ ). As a perturbation method, external pressure can lift the ground state degeneracy and offer detailed information about how the unconventional superconductivity evolve with other electronic orders. For example, an in-plane resistivity anisotropy ${ }^{7}$ and a spin excitation ${ }^{8}$ have been observed in electron-doped $\mathrm{BaFe}_{2} \mathrm{As}_{2}$ under uniaxial pressure; in FeSe with external pressure, the magnetic order could emerge and coexist with the high-temperature superconductivity ${ }^{9}$, while enhanced spin fluctuation is evidenced as well ${ }^{10}$. Among FeSCs, LiFeAs is unique as its phase diagram is not intervened by any magnetic or nematic order, which provides an appropriate platform to detect the relationship between pressure and superconductivity in strong correlated system ${ }^{11,12}$.

Under hydrostatic pressure, transport measurements reveal the transition temperature $T_{\mathrm{c}}$ of LiFeAs declines linearly with increase of pressure amplitude, with no derived order, such as magnetism or nematicity observed ${ }^{11}$. However, when the uniaxial strain is applied to the specific lattice direction, stabilized sematic electronic state emerges, and suppresses the paring strength of superconductivity ${ }^{12}$. These divergent reports demonstrate that, compared with hydrostatic pressure, the orientation-dependent strain would trigger quite different influences on LiFeAs. Given the existence of multiple Fermi surfaces in the Brillouin zone, as well as the physical regime of corelated interactions in this material, the superconducting order parameter affected by orientation-dependent strain are intricate. Therefore, more experimental observations are required.

In this work, by using scanning tunneling microscopy/spectroscopy (STM/S), we report the observation of orientationdependent superconductivity at two types of wrinkles on LiFeAs surface. The tunneling spectra show an increase of superconducting (SC) gaps on type-I wrinkles and a reduction on typeII wrinkles. Compared to wrinkle-free region, temperaturedependent measurements of the SC gap show that the gapclosing temperature on type-I wrinkle region is enhanced by $20-30 \%$, but it remains almost unchanged on type-II wrinkle region. Although wrinkles are a commonly consequence of relieving transverse strain induced by the change of atom coordination ${ }^{13,14}$, the spatial feature of superconducting vortices observed on wrinkles confirms the existence of local strains. While the wrinkle orientations are continuous, the associated SC gap size has an abrupt jump at a certain angle of orientation, which is likely the consequence of Lifshitz transition.

\section{Results}

The wrinkle topography on LiFeAs surface. The atomic model of LiFeAs is shown in Fig. 1a. Unlike many other FeSCs (refs. ${ }^{15,16}$ ), the cleavage of LiFeAs crystal occurs at a nonpolar plane between the two Li layers (dashed line in Fig. 1a), presenting a good platform for investigating and tuning the unconventional superconductivity at the nanoscale ${ }^{17-20}$. The stoichiometric LiFeAs shows superconductivity below the transition temperature $T_{\mathrm{c}}(\sim 17 \mathrm{~K})^{21}$. Two types of wrinkles are observed on LiFeAs surface (Fig. 1b-d), appearing as straight $1 \mathrm{D}$ ridges. Type-I wrinkles extend along the [110] direction (with respect to Li surface, also the Fe-Fe direction) or its neighboring directions, spanning a width about $15 \mathrm{~nm}$ with a maximum height of $\sim 1.0 \AA$ (lower panel, Fig. 1c). Type-II wrinkles extend along the [100] direction (also the Fe-As direction) or its neighboring directions, with a width of $\sim 10 \mathrm{~nm}$ and a maximum height of $\sim 0.7 \AA$ (lower panel, Fig. 1d). Both types of wrinkles are uniform in width and extend from several tens of to hundreds of nanometers. An atomic resolution image of type-I wrinkle in Fig. 1e shows a continuous and perfect Li lattice, excluding the possibility of formation of twin boundary ${ }^{22,23}$, domain wall ${ }^{24}$ or line defects ${ }^{25}$. Within the resolution of STM, no obvious lattice constant change can be detected (Supplemental Fig. 1). We propose that these wrinkles are likely induced by releasing of local strain during the creation of LiFeAs surface upon cleavage. Indeed, by atomic force microscopy (AFM), we explicitly demonstrate that the wrinkles on LiFeAs surface have real spatial corrugations, instead of purely reflecting as enhancement of local density of states (LDOS) (Supplemental Fig. 2).

Local superconductivity tuned by two types of wrinkles. There is remarkable differences between the type-I and II wrinkles in their LDOS. Figure if displays the differential conductance spectra $(\mathrm{d} I / \mathrm{d} V)$ taken at the wrinkle-free region (black curve), type-I (red curve) and type-II wrinkles (blue curve) on the surface, as marked by the crosses in Fig. 1c, d. In the wrinkle-free region, $\mathrm{d} I / \mathrm{d} V$ spectrum shows multigap features of LiFeAs, with a large gap of $\sim 5.8 \mathrm{meV}$ (possibly come from the inner-hole pocket at the $\Gamma$ point) and a small gap of $\sim 2.9 \mathrm{meV}$ (possibly come from the outer-hole pocket at the $\Gamma$ point), which are consistent with previous reports ${ }^{17-19,26}$. Intriguingly, the tunneling spectrum on type-I wrinkle yields a coherence peak at $7.3 \mathrm{meV}$ and a shoulder at $3.6 \mathrm{meV}$, and type-II wrinkle exhibits a single V-shaped gap of $2.5 \mathrm{meV}$. We note that electron doping ${ }^{20,27}$ and application of external pressure ${ }^{11,12}$ normally lead to reduction of SC gap or $T_{c}$, thus the increase of SC gaps is not commonly observed in LiFeAs.

Inspired by the novel gap features observed on the wrinkles, we carry out spatial $\mathrm{d} I / \mathrm{d} V$ spectra line-cut across and along the wrinkles, as displayed in Fig. 2a-d. On a type-I wrinkle, the coherence peaks of $\Delta_{1}$ start to shift to higher energies when getting close to the wrinkle edge and show the constant values of $\pm 7.3 \mathrm{meV}$ across the wrinkle (Fig. 2a). At the same time, the shoulders of $\Delta_{2}$ follow a similar tendency. The enlarged gaps $\Delta_{1}$ and $\Delta_{2}$ remain homogeneous along the type-I wrinkle (Fig. 2b). Furthermore, the gap map of a type-I wrinkle (Supplemental Fig. $3 a, b)$ reveals the maximum gap size $\left(\Delta_{1}\right)$ distribution. It is evident that the type-I wrinkle has larger SC gap sizes compared with the wrinkle-free region. Also, the edges of the wrinkle (Supplemental Fig. 3a, b) show the largest SC gap, possibly suggesting the highest strain at the edges. We compare the SC gaps between the wrinkle-free region and type-I region under different axis scales (Supplemental Fig. 3c). The results show that the enhancement of the two SC gaps is of the same ratio (20-30\%). On a type-II wrinkle, however, the coherence peaks of $\Delta_{1}$ are strongly suppressed when getting close to the wrinkle edge and totally disappear on the wrinkle region. Meanwhile, the shoulders of $\Delta_{2}$ evolve into a pair of coherence peaks on the wrinkle (Fig. 2c). Note that the gap features are robust and homogenous along the type-II wrinkle (Fig. 2d).

We next perform the temperature-dependent $\mathrm{d} I / \mathrm{d} V$ measurements. On a type-I wrinkle, the SC gap $\Delta_{1}$ can be well differentiated at $17 \mathrm{~K}$ ( $T_{\mathrm{c}}$ of bulk LiFeAs) and gradually closes at $\sim 20.5 \mathrm{~K}$ (Fig. 3a), while the gap closes at $17 \mathrm{~K}$ at the wrinklefree region (Fig. 3b). The SC gap of a type-II wrinkle also closes at $\sim 17 \mathrm{~K}$ (Fig. $3 \mathrm{c}$ ). We note that there is a bump near $E_{\mathrm{F}}$ at high temperature (black arrows in Fig. 3a, b), which would be the band top of $d_{\mathrm{xz}}$ (ref. ${ }^{28}$ ). In Fig. $3 \mathrm{~d}$, we plot the extracted gap values as a function of temperature (Supplemental note 11). The wrinkle-free region (black squares) and type-I region (red triangles) follow the same tendency, which is more obvious after rescaling the data of type-I region (pink triangles). This coincidence implies that the 
a

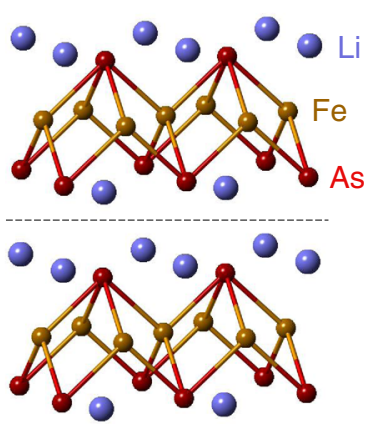

b

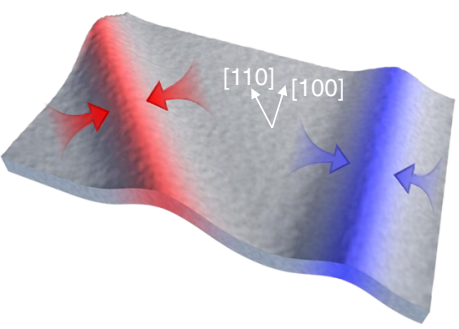

C

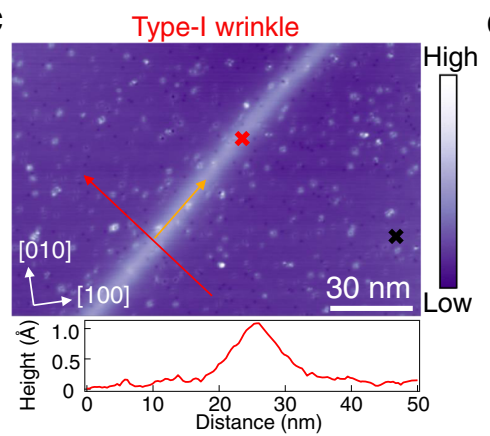

d

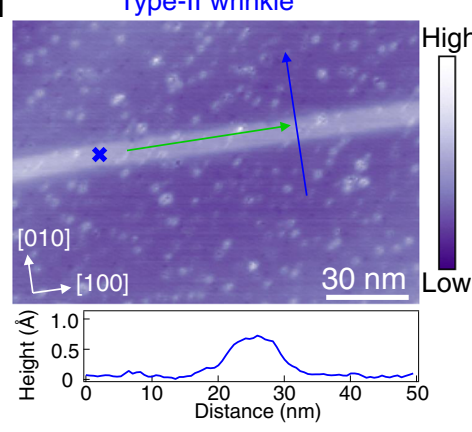

e

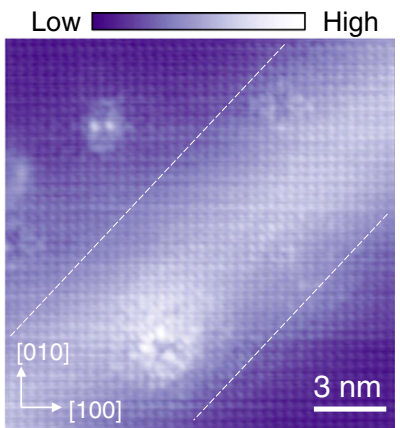

f

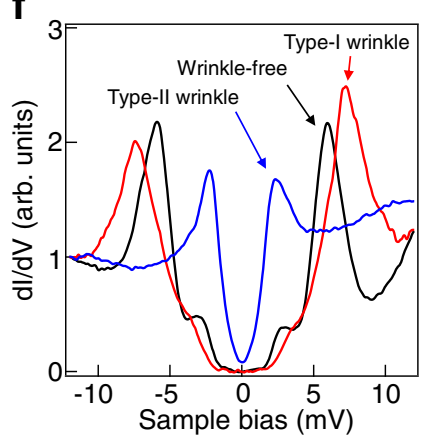

Fig. 1 Strain-induced wrinkles on LiFeAs surface. a The crystal structure of LiFeAs. The black dashed line indicates the location where the cleavage happens. $\mathbf{b}$ The sketches of the two types of wrinkles and the local strain on LiFeAs surface. $\mathbf{c}$ Upper panel: a large scale STM topography of LiFeAs surface showing a type-I wrinkle. The [100] and [010] mark the lattice directions on the Li terminal surface, respectively. Setpoint: $V_{\mathrm{s}}=-20 \mathrm{mV}, I_{\mathrm{t}}=-20 \mathrm{pA}$. Lower panel: a line profile taken along the red line in the upper panel. d Upper panel: a large scale STM topography of LiFeAs surface showing a type-II wrinkle. Setpoint: $V_{\mathrm{s}}=-30 \mathrm{mV}, \mathrm{I}_{\mathrm{t}}=-20 \mathrm{pA}$. Lower panel: a line profile taken along the blue line in the upper panel. e The atomic resolution image of typeI wrinkle. The white dashed line indicates the wrinkle edge. Setpoint: $V_{\mathrm{s}}=-3 \mathrm{mV}, \mathrm{l}_{\mathrm{t}}=-1 \mathrm{nA}$. $\mathbf{f}$ The $\mathrm{d} l / \mathrm{dV}$ spectra taken at the three crosses in (c and $\mathbf{d}$ ). Compared with the SC gaps $\left(\Delta_{1}=5.8 \mathrm{meV}, \Delta_{2}=2.9 \mathrm{meV}\right)$ detected at wrinkle-free region (black curve), two enlarged gaps $\left(\Delta_{1}=7.3 \mathrm{meV}, \Delta_{2}=3.6 \mathrm{meV}\right)$ is observed on type-I wrinkle (red curve), while a smaller gap $\left(2.5 \mathrm{meV}\right.$ ) is observed on type-II wrinkle (blue curve). Setpoint: $V_{\mathrm{s}}=-10 \mathrm{mV}, I_{\mathrm{t}}=-200 \mathrm{pA}$
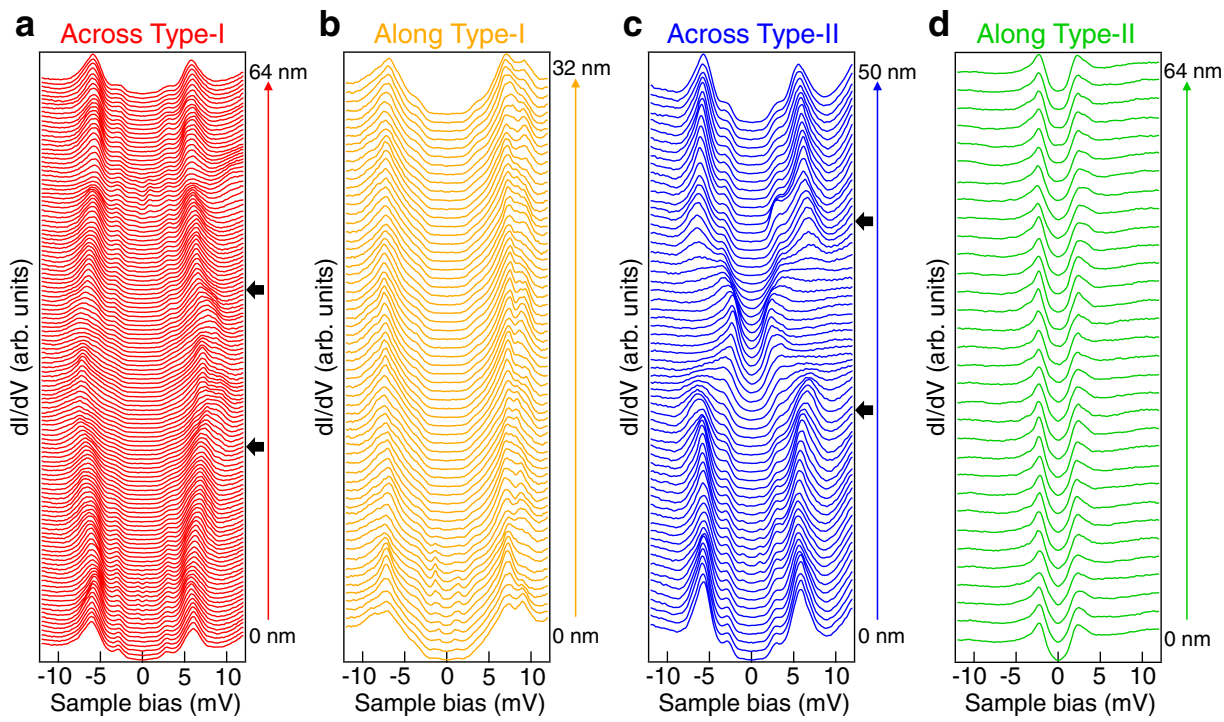

Fig. 2 Superconducting gaps on type-I and type-II wrinkles. $\mathbf{a}$, $\mathbf{b}$ The waterfall plots of $d / / d V$ spectra across (red) and along (orange) the type-I wrinkle as marked by the arrows in Fig. 1c, showing the homogeneous and enlarged superconducting gap along type-I wrinkle. The black arrows in (a) indicate the type-I wrinkle boundary. $\mathbf{c}, \mathbf{d}$ The waterfall plots of $\mathrm{d} / \mathrm{d} \mathrm{d}$ spectra across (blue) and along (green) the type-Il wrinkle as marked by the arrows in Fig. 1d, showing the homogeneous and reduced superconducting gap along type-II wrinkle. The black arrows in (c) indicate the type-II wrinkle boundary. Setpoint for (a-d): $V_{\mathrm{s}}=-10 \mathrm{mV}, \mathrm{I}_{\mathrm{t}}=-200 \mathrm{pA}$. 
a

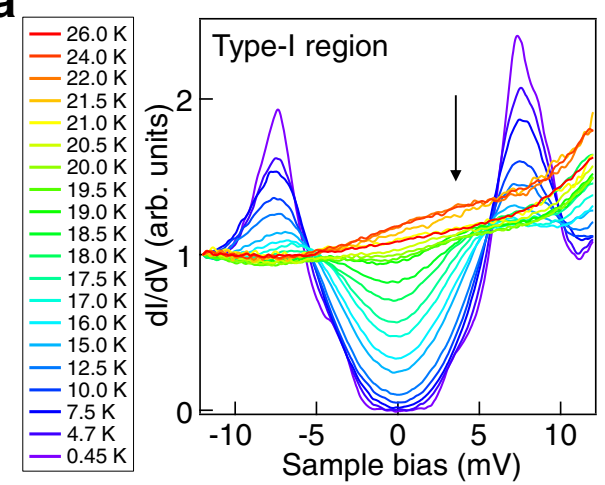

C

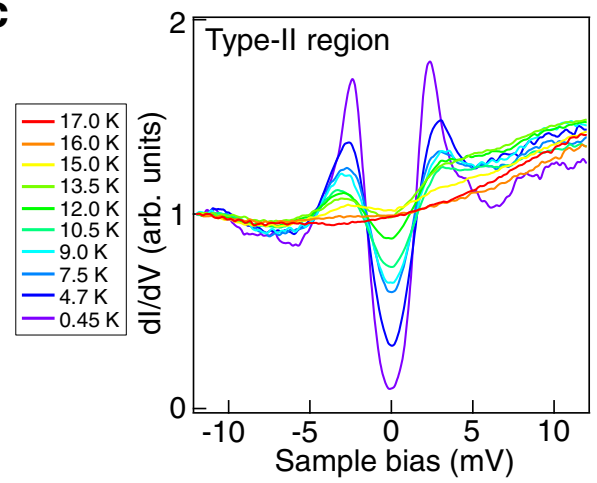

b

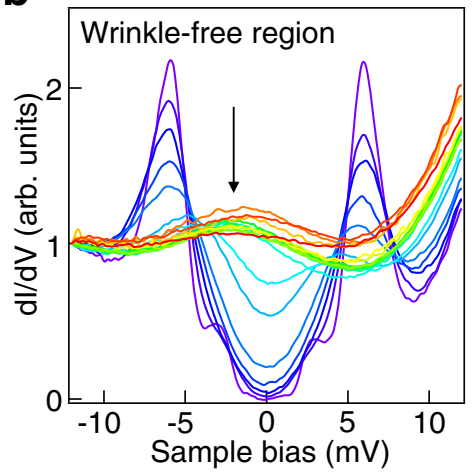

d

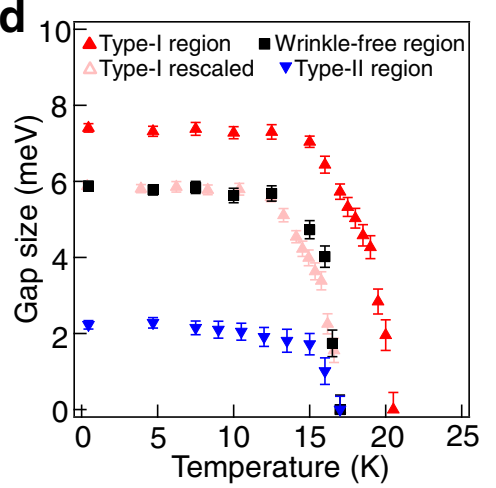

Fig. 3 Temperature dependence of superconducting gaps on the wrinkles. a-c Temperature-dependent $d / / d V$ spectra taken at type-l wrinkle region, wrinkle-free region and type-II wrinkle region, respectively. The black arrows highlight the bump features of the LDOS. (a) and (b) share the same legend. Setpoint: $V_{\mathrm{s}}=-10 \mathrm{mV}, \mathrm{I}_{\mathrm{t}}=-200 \mathrm{pA}$. d A plot of the gap sizes as a function of temperature extracted from a-c

type-I region has similar coupling strength with that of the wrinkle-free region. However, on the type-II region, superconductivity behaves differently.

The $\mathrm{C}_{4}$ symmetry breaking and two distinct superconducting states on wrinkles. To explain the exotic superconducting behaviors we observed, the first step is understanding the circumstances on these wrinkles. Here, we image the vortex structure by zero-bias conductance (ZBC) map (Supplemental Fig. 4). The vortex on the wrinkle-free region present a four-pointed-star like structure due to the $\mathrm{C}_{4}$ symmetry of the Fermi surface of $d_{\mathrm{xy}}$ band ${ }^{29}$ (Fig. 4a). In contrast, the vortex at wrinkles change from $\mathrm{C}_{4}$ symmetric to $\mathrm{C}_{2}$ symmetric shape with the long axis extending along the wrinkle orientation (Fig. 4b). We point out that our vortex structure results not only clarify the existence of local strain on the wrinkles, but also suggests that the configuration of Fermi surface changes consequently.

Next, we plot the statistics of the orientation of the wrinkles and the corresponding SC gaps, which reveals that the [110] direction favors the type-I wrinkles while the [100] direction favors the type-II wrinkles (Supplemental Figs. 9 and 10). Intriguingly, wrinkles along other orientations are also found in our experiment (Fig. 4c, Supplemental Figs. 9 and 10), whereas the gap size versus orientation yields an abrupt change at about $20^{\circ}$ with respect to the [110] direction. The statistical results indicate that the SC gaps do not gradually evolve with wrinkle orientations, but resemble a discontinuous transition (Fig. 4c). We also note that the wrinkles can make turns on the surface, leading to a transition from type-I to type-II (Supplemental Fig. 5).

Further analysis indicates that the switch of the superconductivity on two-type wrinkles is coincidence with the band structure changing under different orientations of local strain.
First, the bump features in Fig. 3a, b indicate the band of $d_{\mathrm{xz}}$ shifts above the Fermi level on the type-I wrinkles (black arrows in Fig. 3a, b). Next, our results indicate that the wrinkles impact the LDOS away from the Fermi level, as well (Fig. 4d). Note that there is a shoulder around $33 \mathrm{meV}$ which has a slight shift comparing to the one in the wrinkle-free region (Supplemental Fig. 6), suggesting the band top of the $d_{\mathrm{xy}}$ orbital shifts up at the type-I wrinkle and down at the type-II wrinkle 28,30 . In addition, the whole gap feature changes from U-shape to $\mathrm{V}$-shape ${ }^{20}$ with nonzero LDOS at the zero energy on the type-II wrinkle (Figs. 1f, 3c, and 4d), and the intensity of LDOS for the type-II wrinkle has a large loss comparing to others as well, both indicate a possibility that the inter-hole bands $\left(d_{\mathrm{xz}}\right.$ and $\left.d_{\mathrm{yz}}\right)$ sink down below the Fermi level on the type-II region.

\section{Discussion}

We note that in previous reports which utilize the external global pressure, only suppressions of superconductivity were found either in the hydrostatic pressure or uniaxial strain, while we simultaneously observed the enhancement and suppression of superconductivity on type-I and type-II wrinkles, respectively. In addition, our results reveal that the unique orientationdependence of gap size separated by an abrupt jump (Fig. 4c), which may suggest the detailed responding mechanism to strain effect is diverse when the strain effect is shrink to nanoscale.

In the Supplemental Table 1, we summarize the superconducting gap size, $T_{\mathrm{c}}$ and the ratio $2 \Delta / k_{\mathrm{B}} T_{\mathrm{c}}$ for wrinkle-free, type-I and type-II wrinkles. The ratios are similar for wrinkle-free and type-I wrinkles, indicating that the paring strength at type-I wrinkle region is close to that at the wrinkle-free region. Therefore, the increase of DOS at type-I wrinkle as proposed here is reasonable. On the other hand, $\Delta_{2}$ at type-II wrinkle is small and the ratio at type-II wrinkle $(\sim 3.4)$ is close to the weak-coupling 

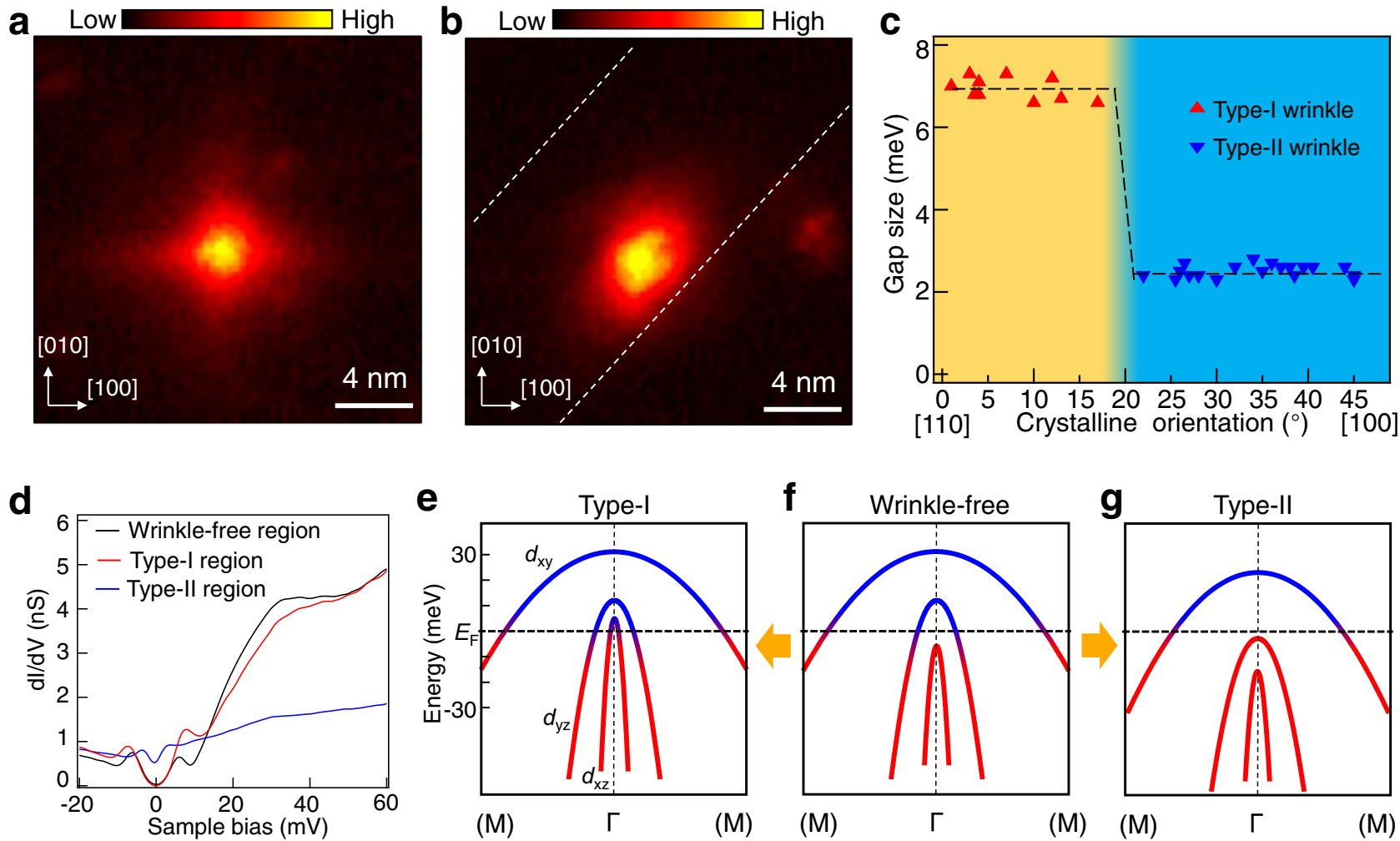

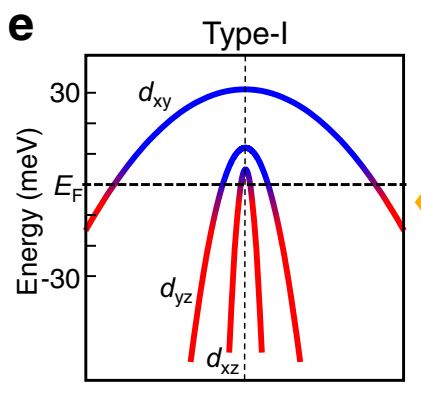

(M)
(M)

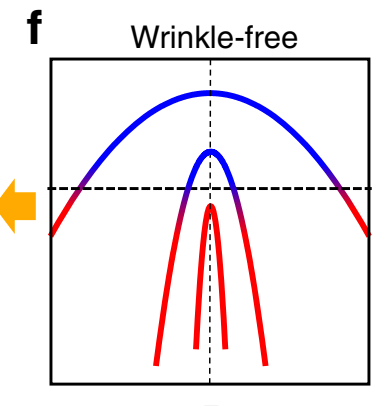

(M)

(M)

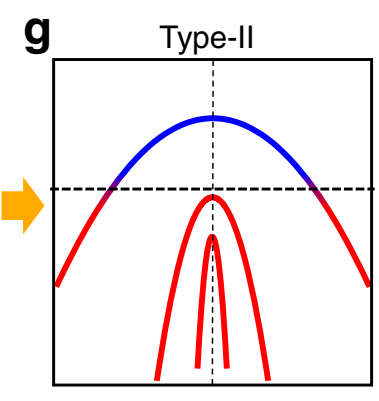

(M)
$\Gamma$

Fig. 4 Uniaxial strain-induced band shifting. a, b Zero-bias conductance map of vortices at wrinkle-free region and type-I wrinkle, respectively. The white dashed line in (b) indicate the wrinkle edge. Setpoint: $V_{\mathrm{s}}=-10 \mathrm{mV}, I_{\mathrm{t}}=-200 \mathrm{pA}$. c Statistics of orientations of the two types of wrinkles. The dashed line is a guide to eye. $\mathbf{d}$ The wide range of $\mathrm{dl} / \mathrm{dV}$ spectra. Setpoint: $V_{\mathrm{s}}=-100 \mathrm{mV}, \mathrm{l}_{\mathrm{t}}=-200 \mathrm{pA}$. The unit for $\mathrm{dl} / \mathrm{dV}$ spectra is set to $\mathrm{nS}$ for the direct comparison of the LDOS at the three regions. $\mathbf{e - g}$ Sketches of the band structures of LiFeAs near the $\Gamma$ point and the band shifting of two types of wrinkles. The energy scale is an estimation, not an accurate value.

BCS regime, suggesting that the $T_{\mathrm{c}}$ at type-II wrinkle may be induced by the bulk superconducting proximity effect. Another possible explanation is that the $d_{\mathrm{xz}}$ as well as $d_{\mathrm{yz}}$ bands sink below $E_{\mathrm{F}}$, and only the smaller gap of $d_{\mathrm{xy}}$ is observed (tunneling at the STM junction is insensitive to the $M$ point), since the gap function in the FeSCs is found to be approximately proportional to $\cos \left(k_{\mathrm{x}}\right) \cos \left(k_{\mathrm{y}}\right)^{31}$. In this way, $T_{\mathrm{c}}$ is not driven by $\Delta_{2}$ which is much smaller than the global superconducting pairing strength.

Based on the observations discussed above, we propose a possible scenario that the local strain on wrinkle causes the shifting of bands around $\Gamma$ point (Fig. $4 \mathrm{e}-\mathrm{g}$ ). In bulk LiFeAs, the $d_{\mathrm{xz}} / d_{\mathrm{yz}}$ bands split possibly due to the spin-orbit coupling ${ }^{28}$, with $d_{\mathrm{yz}}$ crossing $E_{\mathrm{F}}$ and $d_{\mathrm{xz}}$ sinking below (Fig. $4 \mathrm{f}$ ). When local strain exists along the [110] direction (Fe-Fe direction), the $d_{\mathrm{xz}}$ band top shifts above $E_{\mathrm{F}}$, giving rise to an increase of DOS near the $\Gamma$ point (Fig. 4e). The enhanced DOS can increase the SC gap and $T_{\mathrm{c}}$. The same increasing ratio of $\Delta_{1}$ and $\Delta_{2}$ on type-I wrinkles clearly supports this scenario. On the other hand, once the strain is along the [100] direction (Fe-As direction), both the $d_{\mathrm{yz}}$ and $d_{\mathrm{xz}}$ band sink below $E_{\mathrm{F}}$, leaving only the $d_{\mathrm{xy}}$ band crossing $E_{\mathrm{F}}$ (Fig. $4 \mathrm{~g}$ ). In this case, the loss of $d_{\mathrm{yz}}$ Fermi surface leads to the disappearance of the $\Delta_{1}$ gap, and that only the $\Delta_{2}$ gap can be observed, yet the $\mathrm{V}$-shape of the in-gap states is quite puzzling. This V-shape SC gap resembles the tunneling spectra in electron-doped $\mathrm{LiFe}_{1-\mathrm{x}} \mathrm{Co}_{\mathrm{x}}$ As (ref. ${ }^{20}$ ), in which the $E_{\mathrm{F}}$ only passes the $d_{\mathrm{xy}}$ band possibly. We perform density functional theory (DFT) calculations and find the existence of compressive or tensile strain indeed has strong effect on shifting the local $d_{\mathrm{xz}}$ band (Supplemental Fig. 8), which supports the band shifting scenario discussed above. The shifting of bands may induce the possible local
Lifshitz transition ${ }^{32-35}$ at the wrinkle regions. We also note that the superconductivity in LiFeAs might relates to spin fluctuations. It is possible that the As anion height from $\mathrm{Fe}$ atoms ${ }^{36}$ which will be affected by the local strain may modify the spin fluctuations and consequently tune the superconductivity. Recently, a nematicity order is also reported by $\mathrm{STM}^{37}$ and $\mathrm{ARPES}^{38}$ in LiFeAs. In iron-based superconductors, such as $\mathrm{BaFe}_{2} \mathrm{As}_{2}$ (ref. ${ }^{8}$ ) and $\mathrm{Fe}(\mathrm{Te}, \mathrm{Se})\left(\right.$ ref. $\left.{ }^{39}\right)$, a nematicity order emerges under strain. At the wrinkle locations of LiFeAs, the $\mathrm{C}_{4}$ symmetry is broken, suggesting the existence of local strain which may also induce the nematicity order. Therefore, the nematicity order would be another possible origin of our observations. Nevertheless, we cannot rule out other scenarios of superconducting changing 40,41 and further theoretical understanding of the microscopic mechanism is required.

In summary, we have identified two types of wrinkles on LiFeAs surface. The orientation-dependent wrinkles accompanied with local uniaxial strains have very different influence on the superconductivity at the nanoscale, with one gets an enhancement while the other is suppressed. A possible Lifshitz transition scenario is proposed to explain these two distinct states of superconductivity in the vicinity of wrinkles on LiFeAs. Our observations suggest that the change of electronic structure induced by strain has strong influence for unconventional superconductivity in LiFeAs.

\section{Methods}

Single-crystal growth and scanning tunneling microscopy/spectroscopy

(STM/S). High-quality LiFeAs single crystals were synthesized by using the selfflux method ${ }^{27}$. LiFeAs crystals were mounted at a STM sample holder by epoxy in 
a glove box and transferred to a ultra-high vacuum chamber where they were cleaved in-situ, and then immediately transferred to a STM scanner at 4.7 K. STM/ $\mathrm{S}$ measurements were operated at $0.45 \mathrm{~K}$ by ${ }^{3} \mathrm{He}$ single-shot technique (Unisoku). Polycrystal tungsten tips were etched chemically and calibrated on $\mathrm{Au}(111)$ surface before use. All STM images were acquired in the constant-current mode. The differential conductance $(d I / d V)$ spectra and maps were obtained by a standard lock-in amplifier at a frequency of $973.0 \mathrm{~Hz}$, with a modulation voltage of $0.2 \mathrm{mV}$. Magnetic fields were applied perpendicular to the samples.

Noncontact atomic force microscopy (nc-AFM). The nc-AFM measurements were conducted at liquid He temperature with a base ultra-high vacuum lower than $2 \times 10^{-10} \mathrm{mbar}$, where samples were cleaved in-situ. A commercial qPlus tuning fork sensor in frequency modulation mode with $\mathrm{Pt} / \mathrm{Ir}$ tip was used to obtain the data. The resonance frequency was about $27.9 \mathrm{kHz}$ and the stiffness was about $1800 \mathrm{~N} / \mathrm{m}$. The STM topography images were acquired in the constant-current mode. The constant-high and constant-force AFM modes were used to measure the real topography features of two types of wrinkles.

Density functional theory (DFT) calculations. DFT calculations employ the projector augmented wave (PAW) method encoded in the Vienna Ab initio Simulation Package (VASP) ${ }^{42-44}$, and the generalized gradient approximation $(\mathrm{GGA})^{45}$ for the exchange-correlation functional is used. In order to simulate the tensile and compressive strain in the Fe-Fe direction and Fe-As direction respectively without causing a lot of calculations, we have established two different crystal models (Supplemental Fig. 8a, b). We create the supercells which has 120 atoms, including $40 \mathrm{Li}$ atoms, $40 \mathrm{Fe}$ atoms and $40 \mathrm{As}$ atoms. In such a supercell, we stretch or compress one of the $\mathrm{Fe}-\mathrm{Fe}$ bonds (Fe-As bonds), and then use this crystal model to calculate the orbital projected band structure. Although the angle between the two lattices is $45^{\circ}$, the $\mathrm{x}$-axis of the Cartesian coordinate system is set along the FeFe direction in both cases. The calculation parameters we used are as fellow: the plane-wave cut-off energy is $600 \mathrm{eV}$. In self-consistent calculations, $\mathrm{k}$ points are 13 $\times 1 \times 8$ with $\Gamma$ centered. The energy convergence accuracy is $10^{-8} \mathrm{eV}$ in the case of $\mathrm{Fe}-\mathrm{Fe}$ while $10^{-6} \mathrm{eV}$ in the case of Fe-As.

\section{Data availability}

The data in this study are available from the corresponding authors on reasonable request.

Received: 1 June 2021; Accepted: 14 October 2021;

Published online: 02 November 2021

\section{References}

1. Paglione, J. \& Greene, R. L. High-temperature superconductivity in iron-based materials. Nat. Phys. 6, 645-658 (2010).

2. Stewart, G. R. Superconductivity in iron compounds. Rev. Mod. Phys. 83, 1589-1652 (2011).

3. Si, Q., Yu, R. \& Abrahams, E. High-temperature superconductivity in iron pnictides and chalcogenides. Nat. Rev. Mater. 1, 16017 (2016).

4. Hirschfeld, P. J., Korshunov, M. M. \& Mazin, I. I. Gap symmetry and structure of Fe-based superconductors. Rep. Prog. Phys. 74, 124508 (2011).

5. Fernandes, R. M., Chubukov, A. V. \& Schmalian, J. What drives nematic order in iron-based superconductors? Nat. Phys. 10, 97-104 (2014).

6. Fernandes, R. M. \& Chubukov, A. V. Low-energy microscopic models for iron-based superconductors: a review. Rep. Prog. Phys. 80, 014503 (2017).

7. Chu, J. H. et al. In-Plane Resistivity Anisotropy in an Underdoped Iron Arsenide Superconductor. Science 329, 824-826 (2010).

8. $\mathrm{Lu}, \mathrm{X}$. Y. et al. Nematic spin correlations in the tetragonal state of uniaxialstrained $\mathrm{BaFe}_{2-\mathrm{x}} \mathrm{Ni}_{\mathrm{x}} \mathrm{As}_{2}$. Science 345, 657-660 (2014).

9. Sun, J. P. et al. Dome-shaped magnetic order competing with hightemperature superconductivity at high pressures in FeSe. Nat. Commun. 7, 12146 (2016)

10. Sun, J. P. et al. High- $T_{\mathrm{c}}$ superconductivity in FeSe at high pressure: dominant hole carriers and enhanced spin fluctuations. Phys. Rev. Lett. 118, 147004 (2017).

11. Wang, X. C. et al. Effect of pressure on a "111"-type iron pnictide superconductor. High. Press. Res 31, 7-12 (2011).

12. Yim, C. M. et al. Discovery of a strain-stabilised smectic electronic order in LiFeAs. Nat. Commun. 9, 2602 (2018).

13. Edelberg, D. et al. Tunable strain soliton networks confine electrons in van der Waals materials. Nat. Phys. 16, 1097-1102 (2020).

14. Mao, J. et al. Evidence of flat bands and correlated states in buckled graphene superlattices. Nature 584, 215-220 (2020).

15. Hoffman, J. E. Spectroscopic scanning tunneling microscopy insights into Febased superconductors. Rep. Prog. Phys. 74, 124513 (2011).
16. Cao, L. et al. The As-surface of an iron-based superconductor $\mathrm{CaKFe}_{4} \mathrm{As}_{4}$. Nano Res. 14, 3921-3925 (2021).

17. Hanaguri, T. et al. Scanning tunneling microscopy/spectroscopy of vortices in LiFeAs. Phys. Rev. B 85, 214505 (2012).

18. Chi, S. et al. Scanning tunneling spectroscopy of superconducting LiFeAs single crystals: evidence for two nodeless energy gaps and coupling to a bosonic mode. Phys. Rev. Lett. 109, 087002 (2012).

19. Allan, M. P. et al. Anisotropic energy gaps of iron-based superconductivity from intraband quasiparticle interference in LiFeAs. Science 336, 563-567 (2012).

20. Yin, J.-X. et al. Quantum phase transition of correlated iron-based superconductivity in $\mathrm{LiFe}_{1-\mathrm{x}} \mathrm{Co}_{\mathrm{x}}$ As. Phys. Rev. Lett. 123, 217004 (2019).

21. Wang, X. C. et al. The superconductivity at $18 \mathrm{~K}$ in LiFeAs system. Solid State Commun. 148, 538-540 (2008).

22. Song, C. L. et al. Suppression of superconductivity by twin boundaries in FeSe. Phys. Rev. Lett. 109, 137004 (2012).

23. Watashige, T. et al. Evidence for time-reversal symmetry breaking of the superconducting state near twin-boundary interfaces in FeSe revealed by scanning tunneling spectroscopy. Phys. Rev. X. 5, 031022 (2015).

24. Wang, Z. Y. et al. Evidence for dispersing 1D Majorana channels in an ironbased superconductor. Science 367, 104-108 (2020).

25. Chen, C. et al. Atomic line defects and zero-energy end states in monolayer $\mathrm{Fe}(\mathrm{Te}, \mathrm{Se})$ high-temperature superconductors. Nat. Phys. 16, 536-540 (2020).

26. Umezawa, K. et al. Unconventional anisotropic s-wave superconducting gaps of the LiFeAs iron-pnictide superconductor. Phys. Rev. Lett. 108, 037002 (2012).

27. Xing, L. Y. et al. The anomaly $\mathrm{Cu}$ doping effects on LiFeAs superconductors. J. Phys.: Condens. Matter 26, 435703 (2014).

28. Miao, H. et al. Coexistence of orbital degeneracy lifting and superconductivity in iron-based superconductors. Phys. Rev. B 89, 220503(R) (2014).

29. Wang, Y., Hirschfeld, P. J. \& Vekhter, I. Theory of quasiparticle vortex bound states in iron-based superconductors: Application to scanning tunneling spectroscopy of LiFeAs. Phys. Rev. B 85, 020506(R) (2012).

30. Kong, L. Y. et al. Majorana zero modes in impurity-assisted vortex of LiFeAs superconductor. Nat. Commun. 12, 4146 (2021).

31. Richard, P., Qian, T. \& Ding, H. ARPES measurements of the superconducting gap of Fe-based superconductors and their implications to the pairing mechanism. J. Phys. Condens. Matter 27, 293203 (2015).

32. Lifshitz, I. M. Anomalies of electron characteristics of a metal in the high pressure region. Sov. Phys. JEPT 11, 1130-1135 (1960).

33. Liu, C. et al. Evidence for a Lifshitz transition in electron-doped iron arsenic superconductors at the onset of superconductivity. Nat. Phys. 6, 419-423 (2010).

34. Shi, X. et al. Enhanced superconductivity accompanying a Lifshitz transition in electron-doped FeSe monolayer. Nat. Commun. 8, 14988 (2017).

35. Sunko, V. et al. Direct observation of a uniaxial stress-driven Lifshitz transition in $\mathrm{Sr}_{2} \mathrm{RuO}_{4}$. npj Quantum Mater. 4, 46 (2019).

36. Mizuguchi, Y. et al. Anion height dependence of $T_{\mathrm{c}}$ for the Fe-based superconductor. Supercond. Sci. Technol. 23, 054013 (2010).

37. Sun, Z. et al. Spectroscopic evidence of nematic fluctuations in LiFeAs. Phys Rev. B 100, 024506 (2019).

38. Kushnirenko, Y. S. et al. Nematic superconductivity in LiFeAs. Phys. Rev. B 102, 184502 (2020).

39. Zhao, H. et al. Nematic transition and nanoscale suppression of superconductivity in Fe(Te,Se). Nat. Phys. 17, 903-908 (2021).

40. Kang, J., Kemper, A. F. \& Fernandes, R. M. Manipulation of gap nodes by uniaxial strain in iron-based superconductors. Phys. Rev. Lett. 113, 217001 (2014).

41. Huang, G. Q., Xing, Z. W. \& Xing, D. Y. Spin-phonon coupling and effect of pressure in the superconductor LiFeAs: Lattice dynamics from first-principles calculations. Phys. Rev. B 82, 014511 (2010).

42. Kresse, G. \& Hafner, J. Ab initio molecular dynamics for liquid metals. Phys. Rev. B 47, 558-561 (1993).

43. Kresse, G. \& Furthmuller, J. Efficiency of ab-initio total energy calculations for metals and semiconductors using a plane-wave basis set. Comp. Mater. Sci. 6 , 15-50 (1996).

44. Kresse, G. \& Furthmuller, J. Efficient iterative schemes for ab initio total-energy calculations using a plane-wave basis set. Phys. Rev. B 54, 11169-11186 (1996).

45. Perdew, J. P., Burke, K. \& Ernzerhof, M. Generalized gradient approximation made simple. Phys. Rev. Lett. 78, 1396 (1997).

\section{Acknowledgements}

We thank H.M., F.Z., Y.-Y.Z., and G.Q. for helpful discussions. This work is supported by the Ministry of Science and Technology of China (2019YFA0308500, 2018YFA0305700, 2016YFA0401000), the National Natural Science Foundation of China (11888101, 52072401, 61888102, 51991340, 11820101003, 11921004, 11674371), and the Chinese Academy of Sciences (XDB28000000, XDB07000000).

\section{Author contributions}

H.-J.G. and H.D. designed the experiments; L.C., W.L., and G.L. performed the STM experiment with the assistance of S.Z., L.K., and F.Y.; Q.Z., L.H., and X.L. performed the 
AFM experiment; Y.W., K.J. performed the DFT calculations; G.D., X.W., and C.J. synthesized the LiFeAs single crystals; L.C., W.L., G.L., L.K., K.J., W.Z., J.H., H.D., and H.-J.G. analyzed the data with inputs from all other authors; L.C., W.L., G.L., and S.Z. plotted the figures. L.C., W.L., G.L., and K.J. wrote the manuscript with input from all other authors. H.-J.G. and H.D. supervised the project.

\section{Competing interests}

The authors declare no competing interests.

\section{Additional information}

Supplementary information The online version contains supplementary material available at https://doi.org/10.1038/s41467-021-26708-8.

Correspondence and requests for materials should be addressed to Geng Li, Hong Ding or Hong-Jun Gao.

Peer review information Nature Communications thanks Tien-Ming Chuang and the other, anonymous, reviewer(s) for their contribution to the peer review of this work. Peer reviewer reports are available.

Reprints and permission information is available at http://www.nature.com/reprints

Publisher's note Springer Nature remains neutral with regard to jurisdictional claims in published maps and institutional affiliations.

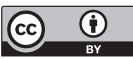
adaptation, distribution and reproduction in any medium or format, as long as you give appropriate credit to the original author(s) and the source, provide a link to the Creative Commons license, and indicate if changes were made. The images or other third party material in this article are included in the article's Creative Commons license, unless indicated otherwise in a credit line to the material. If material is not included in the article's Creative Commons license and your intended use is not permitted by statutory regulation or exceeds the permitted use, you will need to obtain permission directly from the copyright holder. To view a copy of this license, visit http://creativecommons.org/ licenses/by/4.0/.

(C) The Author(s) 2021 